\title{
REDSHIFT SURVEY OF 951 IRAS GALAXIES \\ IN THE SOUTHERN MILKY WAY
}

\author{
N. VISVANATHAN ${ }^{1}$ AND T. YAMADA ${ }^{2}$ \\ ${ }^{1}$ Mount Stromlo and Siding Spring Observatories \\ ${ }^{2}$ Astronomical Institute, Tohoku University
}

\section{Introduction}

In this paper we present the results of a redshift survey of IRAS galaxies behind the whole southern Milky Way region $210^{\circ}<l<360^{\circ}$ at $|b|<15^{\circ}$. The galaxy candidates in the southern Milky Way were selected from IRAS Point Source Catalogue (IPSC) by applying flux density and infrared colour criteria (Strauss et al. 1990; Rowan-Robinson et al. 1991). The selected sample is flux limited with $f_{60}>0.6 J y$. A visual search for galaxy-like objects have been carried out in UK-Schmidt Infrared and IIIaJ films in the selected IRAS positions. In spite of the large Galactic extinction in this region, 966 galaxy candidates have been identified (Yamada et al. 1993).

\section{Redshift Measurements}

Of the 966 galaxy candidates we have 951 confirmed galaxies in the region. Our sample of confirmed galaxies are very incomplete in the inner parts of the galaxy $|b|=5^{\circ}$ and 67 percent complete in the latitude zones, $5^{\circ}<b<$ $15^{\circ}$ and $15^{\circ}<b<-5^{\circ}$. A plot of the distribution of observed velocities of our sample of 951 galaxies in the present survey shows a broad maximum between 3000 to $6000 \mathrm{~km} \mathrm{~s}^{-1}$ which can be taken to mean the survey is nearly complete up to $5000 \mathrm{~km} \mathrm{~s}^{-1}$. Also a long tail is seen extending to $20,000 \mathrm{~km} \mathrm{~s}^{-1}$ which is due to decreasing incompleteness of our sample at larger redshifts. It is interesting that the IRAS galaxy sample outside the Milky Way for $f_{60}>0.6 J y$ shows a peak around $5000 \mathrm{~km} \mathrm{~s}^{-1}$ (RowanRobinson et al. 1991). 


\section{Discussion}

Cone diagrams exhibiting the detailed distribution of all the identified IRAS galaxies with $v<10,000 \mathrm{~km} \mathrm{~s}^{-1}$ in the region $210^{\circ}<l<360^{\circ}$ give the following picture. In negative latitudes near $l=240^{\circ}$ at $\mathrm{v}=2500 \mathrm{~km} \mathrm{~s}^{-1}$, the Puppis cluster is identified. The best estimate of the position of the cluster is $l=240^{\circ} \mathrm{b}=-7^{\circ}$, and its redshift is $2400 \mathrm{~km} \mathrm{~s}^{-1}$. At $\mathrm{l}=245^{\circ}$, $\mathrm{b}=-5^{\circ}$ a new concentration of galaxies is visible at $7500 \mathrm{~km} \mathrm{~s}^{-1}$. Also a void is seen at $l=240^{\circ}$ to $270^{\circ}, \mathrm{v}=2500$ to $3500 \mathrm{~km} \mathrm{~s}^{-1}$. In positive latitudes at $270^{\circ}<l<360^{\circ}, v<5000 \mathrm{~km} \mathrm{~s}^{-1}$ the extension of the Hydra, Centaurus ( both Hi and Lo velocity components ) and Antlia clusters are seen. A clump of galaxies is also seen in the positive latitudes near 1 $=280^{\circ}, \mathrm{v}=5500 \mathrm{~km} \mathrm{~s}^{-1}$. Further the rich cluster A3627 $\left(\mathrm{l}=325^{\circ}, \mathrm{b}=-7^{\circ}\right.$, $\mathrm{v}=4500 \mathrm{~km} \mathrm{~s}^{-1}$ ) studied in detail by Kraan-Kortewag et al. (1996) could be identified. The GA region $270^{\circ}<l<350^{\circ}$ at $|b|<15^{\circ}$ is dominated by overdensity of galaxies representing the extension of Hydra-Centaurus complex in the positive latitudes and the Pavo-Indus complex in the negative latitudes. The broad over density of galaxies seen in our data corresponds with the distant concentration seen at $4500 \mathrm{~km} \mathrm{~s}^{-1}$ in the Supergalactic Plane survey (Dressler 1991) that covers the same range in longitudes as us, but a larger range in latitude $\mathrm{b}\left(-30^{\circ}\right.$ to $-10^{\circ}$ and $+10^{\circ}$ to $\left.+45^{\circ}\right)$. We conclude that the overdensity seen in our survey connects the Centaurus-Hydra complex $\left(\mathrm{l}=302^{\circ}, \mathrm{b}=-22^{\circ}\right)$ to the major concentration Pavo Supercluster $\left(l=332^{\circ}, b=-24^{\circ}\right)$ through the Milky Way. Thus our study strengthens the conclusion of the previous surveys (Dressler 1991; Strauss et al. 1990; Visvanathan 1994; Fisher et al. 1995) that this whole region of increased density is responsible for the peculiar velocity field observed in the local region. Also the centroid of this extensive concentration could be situated at the Milky Way itself $\left(\mathrm{l}=320^{\circ}, \mathrm{b}=0^{\circ}\right)$ at $\mathrm{v}=4500 \mathrm{~km} \mathrm{~s}^{-1}$. Support for our conclusion comes from Kolatt et al. (1995) who finds a peak in massdensity in the Potent reconstruction of peculiar velocity data at $l=320^{\circ}$, $\mathrm{b}=0^{\circ}$ at $\mathrm{v}=4000 \mathrm{~km} \mathrm{~s}^{-1}$.

\section{References}

Dressler, A., 1991, Astrophys.J.Suppl., 75, 241

Fisher,K.B. et al., 1995, Astrophys.J.Suppl., 100, 70

Fisher,K.B. et al., 1995, Astrophys.J.Suppl., 100, 70

Kolatt, T., Dekel, A. \&Lahav, O., 1995, Mon.Not.R.astron.Soc., 275, 797

Kraan-Korteweg, R.C. et al., 1996, Nature, 379, 519

Rowan-Robinson, M., et al., 1991, Mon.Not.R.astron.Soc., 253, 485

Strauss, M.A., et al., 1990, Astrophys.J., 361, 49.

Visvanathan, N.,1994, in "Cosmic velocity fields," ed F.R. Bouchet and M. Lachiez-Rey, editions Frontiers, 125

Yamada, T., et al., 1993, Astrophys.J.Suppl., 89, 57 